\title{
Dilatação do tempo, referenciais acelerados e o paradoxo dos gêmeos
}

Time dilation, accelerated frames, and the twin paradox

\author{
Gabriel B.R.L. de Freitas ${ }^{1}$, André H. Gomes ${ }^{* 10}$
}

${ }^{1}$ Universidade Federal do Espírito Santo, Departamento de Ciências Naturais, Campus São Mateus, Vitória, ES, Brasil

Recebido em 12 de Outubro, 2018. Revisado em 12 de Novembro, 2018. Aceito em 13 de Novembro, 2018.

\begin{abstract}
Ainda que o problema do paradoxo dos gêmeos seja tão antigo quanto à própria teoria da relatividade especial, a compreensão da sua resolução é, muitas vezes, obscurecida por falhas na intuição do estudante. Geralmente, estas se originam em um entendimento incompleto das previsões da teoria ou do seu limite de validade. Neste artigo, apresentamos uma resolução didática do problema dos gêmeos com intuito de elucidar algumas dessas falhas. Dando ênfase ao real conteúdo da previsão relativística de dilatação do tempo, eliminamos uma primeira intuição que possa sugerir a existência de um paradoxo. A partir disso, mostramos como a relatividade especial é suficiente para abordar o problema, não havendo necessidade de recorrer à relatividade geral. Finalizamos apresentando uma resolução do problema considerando tanto do ponto de vista do gêmeo estacionário quanto o do gêmeo viajante. Palavras-chave: Paradoxo dos gêmeos, dilatação do tempo, relatividade especial.
\end{abstract}

\begin{abstract}
Although the twin paradox is as old as the special theory of relativity itself, understanding of its resolution is often obscured by student's miscarried intuition. That is usually a result of an incomplete understanding of some predictions of the theory or of its range of validity. In this article, we present a didactic solution to the problem aiming at elucidating some understanding gaps. Discussing the precise content of the time dilation predicted by special relativity, we eliminate a first intuition that might suggest the existence of a paradox. Then we show why special relativity alone is enough to solve the problem without any need to invoke the general theory of relativity. We conclude this work presenting a solution to the twin paradox from the point of view of the stationary twin as well as from the traveling twin.
\end{abstract}

Keywords: Twin paradox, time dilation, special relativity.

\section{Introdução}

O problema do "paradoxo" do gêmeos é bastante popular no contexto da teoria da relatividade especial (TRE), sendo formulado das mais diversas maneiras. De modo simples, o problema envolve dois gêmeos, Ana e Bob, que estão inicialmente juntos em repouso relativo. Enquanto Ana permanece estacionária, Bob sai em viagem pelo espaço e retorna anos depois. De acordo com a dilatação do tempo prevista pela TRE, no reencontro, os gêmeos devem constatar que Bob envelheceu menos que Ana. Apesar de dramática, essa conclusão já não é mais surpresa. O problema fica interessante quando imaginamos a mesma situação do ponto de vista de Bob. Para ele, Ana é o gêmeo viajante, que vai embora e depois volta, enquanto ele que é o gêmeo estacionário. Como fica a conclusão anterior? Ela inverte-se, sugerindo que Ana envelhece menos que Bob? Qualquer suspeita de resposta positiva caracterizaria o paradoxo que dá nome a este problema. Logo, o problema em questão é entender porque a resposta a estas perguntas tem que ser $n \tilde{a} o$.

*Endereço de correspondência: andre.h.gomes@ufes.br
Geralmente, a intuição física que sugere uma resposta positiva surge de um entendimento incompleto da previsão que a TRE faz para a dilatação do tempo. Quando tal intuição é seguida, a controvérsia em torno do problema dos gêmeos é, por vezes, realçada pela noção errônea de que a TRE é incapaz de lidar com situações que envolvem acelerações, sendo, por isso, necessário abordar o problema utilizando a relatividade geral. Essas são confusões que surgiram e foram resolvidas ao longo das primeiras décadas após a publicação do artigo onde Einstein apresenta a TRE [1] 1] mas que, em diferentes graus, persistem até hoje entre estudantes ${ }^{2}$ Desde então, inúmeros artigos dedicaram-se a esclarecer onde a intuição erra e a como explicar a razão de Bob também concluir que Ana, de fato, envelhece mais que ele ao fim da jornada [5].

Não é nosso objetivo fazer uma revisão das várias abordagens ao problema - para isso, o artigo $[5]$ faz um ótimo trabalho - mas, sim, utilizar o problema dos gêmeos

\footnotetext{
${ }^{1}$ Traduzido para o português em 2 (p. 143) sob o título Sobre a eletrodinâmica dos corpos em movimento.

${ }^{2}$ Os motivos dessa persistência são diversos e fogem ao escopo deste trabalho. Ainda assim, vale mencionar que alguns destes carregam a história da origem e popularização do problema do paradoxo dos gêmeos 3 4.
} 
para esclarecer porque a previsão de dilatação do tempo não conduz à conclusões paradoxais e, em particular, ilustrar como a TRE lida com movimentos acelerados. Sendo a teoria formulada em termos de referenciais inerciais e das transformações que os relacionam, durante o aprendizado nem sempre é claro como ela pode ser utilizada, por exemplo, para descrever o movimento de partículas aceleradas. O que costuma ser ainda menos claro é como fazer previsões em referenciais não-inerciais utilizando a TRE ou, até mesmo, se ela é capaz disso. Para esclarecer estes pontos, resolveremos o problema dos gêmeos utilizando a TRE para determinar as previsões, tanto do gêmeo inercial (Ana) quanto do não-inercial (Bob), para os tempos que cada um registra até o reencontro.

Apesar do claro intuito didático, nosso trabalho também é motivado pela resolução do problema por si só. Em particular, formularemos o problema com Ana em repouso e Bob em movimento hiperbólico ${ }^{3} \mathrm{O}$ fato deste tipo de movimento ser estudado na maioria dos cursos introdutórios de TRE torna nossa abordagem atraente pela simplicidade matemática e por, ao mesmo tempo, preservar a riqueza conceitual envolvida com o movimento acelerado de Bob. Dentre outros, isso facilitará o entendimento das previsões feitas no referencial não-inercial de Bob, que é onde reside o maior desafio à compreensão completa da resolução do problema.

Na próxima seção, faremos uma revisão da TRE com foco na interpretação da dilatação do tempo prevista pela teoria e discutiremos o que é necessário para estender tal previsão para situações envolvendo movimentos acelerados. A resolução do problema dos gêmeos será feita, na Seção 3 segundo o ponto de vista de Ana e, na Sec. 4 segundo o de Bob. Na Sec. 5 apresentaremos os comentários finais a respeito deste trabalho.

\section{Relatividade Especial}

\subsection{Definições auxiliares}

Podemos dizer que as previsões cinemáticas da TRE para o comportamento de réguas e relógios constituem a base sobre a qual todas as outras previsões são construídas, pois é com réguas e relógios que construímos referenciais para medidas de posição e tempo. A interpretação correta destas previsões dependem, portanto, de uma definição adequada do que é um referencial e de como tais medidas são realizadas. Seguiremos as definições apresentadas em [8], as quais resumimos a seguir. Por simplicidade, toda discussão a seguir será feita no contexto de um espaçotempo com somente uma dimensão espacial.

Primeiramente, definimos os chamados relógios-padrão. Estes são relógios idealizados, infinitesimais, todos construídos da mesma maneira, que satisfazem a hipótese do

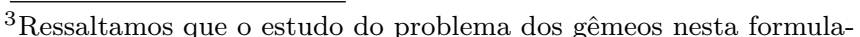
ção não é algo inédito — por exemplo, também foi feito em 6. 7 . - mas, dentro do que constatamos, a maneira como abordamos sua resolução é nova na literatura.
}

relógio ${ }^{4}$ Segundo esta, quaisquer dois relógios que, em um certo instante $t$, possuem a mesma velocidade $v(t)$, registram o mesmo intervalo infinitesimal de tempo $d t$ entre $t$ e $t+d t$, independente da aceleração que cada um tenha. De modo similar, as réguas-padrão são réguas infinitesimais idênticas tais que qualquer par que possua a mesma velocidade $v(t)$ em um instante $t$ possuirá o mesmo comprimento $L(t)$ naquele instante, independente de suas acelerações. Daqui em diante, por régua e relógio, estaremos sempre nos referindo às réguas e relógios padrões.

Para o processo de medida de posição e tempo de um evento qualquer, assumimos ter disponível um número infinito de réguas e relógios. Em particular, tais medidas são realizadas por entidades idealizadas chamadas observadores: partículas pontuais inteligentes, equipadas com um relógio e uma régua, cuja interação com o meio pode ser desprezada.

Para definirmos como são feitas medidas de posição e tempo, definimos um referencial rígido como sendo um conjunto contínuo de infinitos observadores, posicionados lado a lado, um em repouso relativo ao outro, preenchendo todo o espaço, todos com seus relógios perfeitamente sincronizados e réguas perfeitamente alinhadas.

Em um referencial rígido, as linhas de mundo de todos os observadores que o compõem são sempre paralelas. Portanto, a localização de um evento no espaçotempo pode ser estabelecida sabendo-se a posição do observador junto ao qual o evento ocorreu e a posição na linha de mundo deste observador (registrada por seu relógio) correspondente a quando este evento ocorreu. Em particular, a medida do intervalo de tempo entre dois eventos que ocorrem em pontos diferentes do espaço exige o uso de dois relógios, pois o instante em que cada evento ocorre é registrado por um observador diferent $\oint^{5}$ (Fig. 1a). Por outro lado, eventos que ocorrem no mesmo local, mas em instantes diferentes, são registrados por somente um observador e seu relógio (Fig. [1b).

\subsection{Os dois postulados fundamentais}

A teoria da relatividade especial baseia-se nos seguintes dois postulados [1]:

(i) As leis da física são as mesmas em todos referenciais inerciais (princípio da relatividade).

(ii) A luz se propaga de modo retilíneo no vácuo com velocidade constante $c$ em todos os referenciais inerciais (constância da velocidade da luz).

\footnotetext{
${ }^{4}$ Mais detalhes a respeito desta hipótese na Sec. 2.4

5 "Medir", segundo a definição que apresentamos, é o que, ao longo deste trabalho, também chamaremos de observar. Este deve ser diferenciado do ato de ver, que é executado sempre por um único observador 9]. O registro das coordenadas de um evento visto depende do tempo que a luz gasta para ir do ponto de ocorrência do evento até o ponto onde está o observador que o vê. Para um evento observado, o registro corresponde exatamente às coordenadas de posição e tempo da ocorrência do evento.
} 


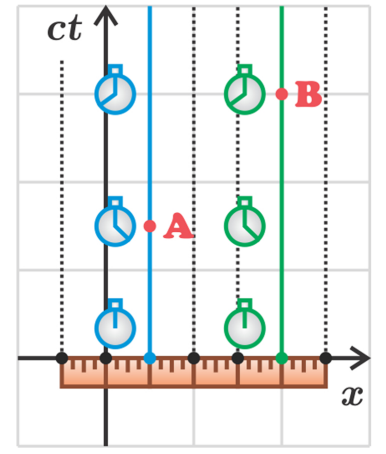

(a)

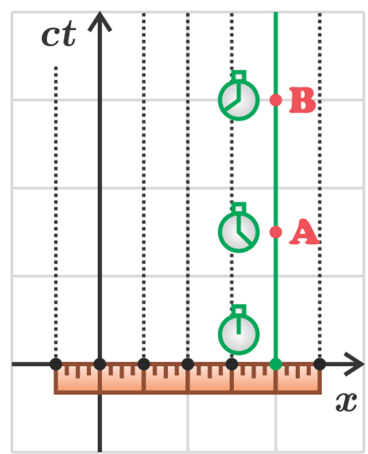

(b)
Figura 1

Mesmo no contexto da TRE, os referenciais inerciais ainda podem ser definidos como aqueles onde a primeira lei de Newton é válida, mas agora englobando algo mais: também são aqueles onde a luz se propaga de modo retilíneo e com velocidade constante $c$ 10. Embutida nestes dois postulados, está a hipótese de homogeneidade e isotropia do espaçotempo. Levando-se em conta tal hipótese de modo explícito, a partir dos postulados verifica-se que o conjunto de transformações de coordenadas que relaciona dois referenciais inerciais são as chamadas transformações de Lorentz 11. ${ }^{6}$ Isso permite reescrever o primeiro postulado como "as leis da física devem ser invariantes sob transformações de Lorentz".

Para estabelecer nossa notação, consideremos o caso particular de dois referenciais inerciais com orientações espaciais idênticas: o referencial $\mathcal{S}$ de coordenadas $(x, y, z, t)$ e o $\mathcal{S}^{\prime}$ de coordenadas $\left(x^{\prime}, y^{\prime}, z^{\prime}, t^{\prime}\right)$, cujas origens coincidem no que ajustamos ser o instante zero tanto para os relógios de $\mathcal{S}$ quanto para os de $\mathcal{S}^{\prime}$. O referencial $\mathcal{S}^{\prime}$ está em movimento retilíneo uniforme (MRU) com velocidade $\boldsymbol{v}$ relativa a $\mathcal{S}$ no sentido positivo do eixo $x$. Nesta situação, as coordenadas destes referenciais para um certo evento no espaçotempo se relacionam pelas seguintes transformações de Lorentz:

$$
\begin{array}{ll}
c t^{\prime}=\gamma(c t-v x / c), & x^{\prime}=\gamma(x-v t), \\
y^{\prime}=y, & z^{\prime}=z,
\end{array}
$$

onde $\gamma \equiv 1 / \sqrt{1-v^{2} / c^{2}}$ é o chamado fator de Lorentz..$^{7}$ As transformações inversas, que levam das coordenadas

\footnotetext{
${ }^{6}$ Para sermos exatos, o conjunto completo de transformações de coordenadas que respeita os dois postulados da TRE e suas hipóteses internas são as chamadas transformações de Poincaré, que adicionam às transformações de Lorentz também rotações espaciais e translações no espaço e tempo [12. Para o que buscamos com este trabalho, considerar as transformações de Lorentz, somente, é o bastante.

${ }^{7}$ Transformações para referenciais em movimento relativo em direções quaisquer podem ser obtidas por generalização direta deste caso particular — veja, por exemplo, a Sec. 6.4 de [13.
}

de $\mathcal{S}^{\prime}$ para as de $\mathcal{S}$, são

$$
\begin{array}{ll}
c t=\gamma\left(c t^{\prime}+v x^{\prime} / c\right), & x=\gamma\left(x^{\prime}+v t^{\prime}\right), \\
y=y^{\prime}, & z=z^{\prime} .
\end{array}
$$

A relação entre as transformações de Lorentz e suas inversas ilustra bem a equivalência entre referenciais inerciais, pois as inversas podem ser obtidas de modo imediato a partir das transformações de $\mathcal{S}$ para $\mathcal{S}^{\prime}$ trocando-se $(x, y, z, t)$ por $\left(x^{\prime}, y^{\prime}, z^{\prime}, t^{\prime}\right)$, e vice-versa, e $v$ por $-v$.

Neste trabalho, por simplicidade, consideraremos apenas movimentos unidimensionais ao longo do eixo $x$, logo omitiremos as coordenadas $y$ e $z$. Aqui, o referencial denotado por $\mathcal{S}$ será sempre o referencial inercial em relação ao qual outros referenciais se movem, sendo estes outros denotados por $S^{\prime}$ caso também sejam inerciais ou por $\overline{\mathcal{S}}$ caso sejam não-inerciais. O par de coordenadas que localiza um evento em cada um destes referenciais será $(x, t),\left(x^{\prime}, t^{\prime}\right)$ e $(\bar{x}, \bar{t})$, respectivamente.

\subsection{Dilatação do tempo entre referenciais inerciais}

Nesta seção, faremos uma discussão cuidadosa da dilatação do tempo prevista a partir dos dois postulados fundamentais da TRE 8 É um mal entendimento desta que sugere a existência de um paradoxo no problema do gêmeos - exatamente o que queremos evitar. No que se segue, estaremos sempre falando de um observador do referencial $\mathcal{S}$ e outro do $\mathcal{S}^{\prime}$, estando $\mathcal{S}^{\prime}$ em MRU com velocidade $v$ relativa a $\mathcal{S}$.

Primeiramente, devemos enunciar outra previsão da TRE: a relatividade da simultaneidade. Esta diz que eventos que são simultâneos em um referencial inercial $\mathcal{S}$ não o serão em um outro referencial inercial $\mathcal{S}^{\prime}$ em movimento relativo. Visto de outra maneira, esta previsão diz que relógios sincronizados no referencial $\mathcal{S}$ não exibem sincronismo do ponto de vista dos observadores do referencial $\mathcal{S}^{\prime}$, e vice-versa.

Finalmente, para discutir a dilatação do tempo de modo preciso, vamos considerar a seguinte situação: uma partícula instável é colocada em repouso no referencial $\mathcal{S}^{\prime}$. Sejam dois eventos: um no instante $t_{1}^{\prime}$, caracterizado pela partícula ser posicionada em repouso na posição $x^{\prime}$, e o outro no instante posterior $t_{2}^{\prime}$, quando a partícula decai. Por ambos eventos ocorrerem em uma mesma posição $x^{\prime}$ em $\mathcal{S}^{\prime}$, falamos que o relógio neste ponto registra o tempo próprio $\tau^{\prime}$ associado à ocorrência destes eventos, fornecendo o intervalo de tempo próprio $\Delta \tau^{\prime}=$ $t_{2}^{\prime}-t_{1}^{\prime}$. Mais importante, somente um relógio (este em $\left.x^{\prime}\right)$ é necessário para fazer tal medição. No referencial $\mathcal{S}$, que observa $\mathcal{S}^{\prime}$ em MRU, a situação é outra, pois os dois eventos ocorrem em pontos distintos, $x_{1}$ e $x_{2}$, em

\footnotetext{
${ }^{8}$ Deduções das principais previsões cinemáticas da TRE - relatividade da simultaneidade, dilatação do tempo e contração do comprimento - podem ser vistas, por exemplo, no Cap. 12 de 14]. Julgamos especialmente enriquecedoras as discussões feitas em 15 por meio de diagramas de espaçotempo.
} 
instantes distintos, $t_{1}$ e $t_{2}$, respectivamente, exigindo dois relógios (um em $x_{1}$ e outro em $x_{2}$ ) para fazer a medição do intervalo $\Delta t=t_{2}-t_{1}$. A chamada dilatação do tempo, prevista pela TRE, diz que a razão entre o intervalo de tempo $\Delta t$ e o intervalo de tempo próprio $\Delta \tau^{\prime}$ é igual a $\gamma$, isto é,

$$
\frac{\Delta t}{\Delta \tau^{\prime}}=\gamma=\frac{1}{\sqrt{1-v^{2} / c^{2}}}>1
$$

Baseando-se na situação descrita, um observador do referencial $\mathcal{S}$ afirmaria que o tempo experimentado por observadores do referencial $\mathcal{S}^{\prime}$ passa de modo mais lento do que o seu.

Veja que a situação que descrevemos não é simétrica: enquanto em $\mathcal{S}^{\prime}$ somente um relógio (em $x^{\prime}$ ) é suficiente para medir $\Delta \tau^{\prime}$, em $\mathcal{S}$ são necessários dois (um em $x_{1}$ e outro em $x_{2}$ ) para medir $\Delta t$. Portanto, a conclusão anterior não pode ser simplesmente invertida para o ponto de vista do observador em $\mathcal{S}^{\prime}$, isto é, $\Delta \tau^{\prime} / \Delta t \neq \gamma$.

Por outro lado, em uma situação análoga, mas $d i$ ferente, podemos imaginar uma partícula instável em repouso no referencial $\mathcal{S}$ (ao invés de $\mathcal{S}^{\prime}$ ) e considerar dois eventos semelhantes ao do caso anterior. Neste caso, a medição de tempo em $\mathcal{S}$ requer somente um relógio para registrar o intervalo de tempo próprio $\Delta \tau$ entre os eventos (que, neste caso, ocorrem no mesmo ponto $x$ ). Diferente do caso anterior, agora $\mathcal{S}^{\prime}$ observa o decaimento ocorrer em dois pontos diferentes, $x_{1}^{\prime}$ e $x_{2}^{\prime}$, logo precisa de dois relógios para registrar os eventos que ocorrem nos instantes $t_{1}^{\prime}$ e $t_{2}^{\prime}$, respectivamente. De acordo com a dilatação do tempo, a razão entre o intervalo de tempo $\Delta t^{\prime}=t_{2}^{\prime}-t_{1}^{\prime}$ e o intervalo de tempo próprio $\Delta \tau$ é

$$
\frac{\Delta t^{\prime}}{\Delta \tau}=\frac{1}{\sqrt{1-v^{2} / c^{2}}}>1 .
$$

Portanto, contrário à conclusão da situação anterior, o observador em repouso junto a $\mathcal{S}^{\prime}$ afirmaria que é o tempo experimentado pelos observadores em movimento junto a $\mathcal{S}$ que passa de modo mais lento.

Como isso é possível? Como conciliar essas conclusões aparentemente contraditórias? De imediato, devemos enfatizar que não há qualquer contradição entre as expressões (5) e (6), pois $\Delta t \neq \Delta \tau$ e $\Delta t^{\prime} \neq \Delta \tau^{\prime}$ uma vez que essas desigualdades envolvem medidas realizadas de modo diferente (uso de um relógio vs. uso de dois relógios) de eventos diferentes (decaimento de uma partícula em repouso em $\mathcal{S}$ vs. decaimento de uma partícula em repouso em $\mathcal{S}^{\prime}$ ). Finalmente, ainda que as expressões (5) e (6) sugiram conclusões contraditórias, ambas estão corretas dos seus respectivos pontos de vista. A chave para compreender isso é lembrar que referenciais são construídos com base no sincronismo de relógios e que a relatividade da simultaneidade impõe que relógios sincronizados em um referencial não mantêm sincronia quando observados de outro referencial em movimento relativo. Com isso em mente, temos que um observador em $\mathcal{S}$ tem como conclusão a expressão (5) e julga que a expressão (6) é inválida, pois, para ele, a medição de $\Delta t^{\prime}$ em $\mathcal{S}^{\prime}$ utilizou dois relógios fora de sincronia. Por outro lado, um observador de $\mathcal{S}^{\prime}$ diz que somente (6) está correta e que (5) é invalidada pelo observador de $\mathcal{S}$ não ter usado relógios sincronizados para medir $\Delta t$. Portanto, neste tipo de situação, não existe uma resposta adequada para perguntas do tipo "para quem o tempo passa mais devagar?" pois não é possível comparar as conclusões de observadores dos dois referenciais.

Caso um observador de $\mathcal{S}$ se chamasse Ana e um de $\mathcal{S}^{\prime}$ se chamasse Bob, e ambos fossem gêmeos, cada um registraria intervalos de tempo indicando que o outro envelhece mais lentamente e ambos estariam corretos dos seus respectivos pontos de vista. Não haveria qualquer paradoxo, pois um julgaria que os registros do outro estariam comprometidos pelo uso de relógios fora de sincronia, e vice-versa. Esta situação jamais se modificaria caso eles se mantivessem, sempre, em MRU relativo. Somente se um deles, ou ambos, alterar o seu movimento para permitir um reencontro é que seria possível comparar qual envelheceu mais.

Com esta discussão, vemos como a relatividade da simultaneidade pode impedir conclusões absolutas a respeito de medições de intervalo de tempo em diferentes referenciais. Por outro lado, comparações de intervalos de tempo próprio não sofrem desta dificuldade, pois demandam o uso de somente um relógio em cada referencial. Como vimos, esta comparação não pode ser feita entre referenciais em MRU relativo, já que, neste caso, os tempos próprios medidos estão associados a pares de eventos diferentes. Tal comparação é possível somente se o intervalo de tempo registrado for entre dois eventos que ocorrem no mesmo ponto espacial em ambos referenciais, pois isso garante que somente um relógio de cada referencial será necessário para tal medição. Para isso acontecer, pelo menos um deles deve estar em um movimento acelerado que, em algum instante, inverte de sentido: o primeiro evento será o primeiro encontro entre os dois referenciais e o segundo evento será o segundo encontro. Esta é, basicamente, a situação presente no problema dos gêmeos descrito na introdução deste artigo.

Como apresentados na Sec. 2.2, os dois postulados da TRE não são suficientes para discutir situações em que um dos referenciais está acelerado, pois dizem respeito somente a referenciais inerciais. Como veremos na próxima seção, a utilização da chamada hipótese do relógio - introduzida de modo implícito por Einstein no artigo em que apresenta a TRE - é suficiente para estender o arcabouço da teoria para lidar com movimentos acelerados.

\subsection{Postulado extra: a hipótese do relógio}

Originalmente, Einstein utilizou os dois postulados apresentados na Sec. 2.2 para deduzir a dilatação do tempo, dada pela nossa Eq. (5). Ele faz isso na Seção I.4 do artigo em que introduz a TRE [1], a qual encerra dizendo que 
esta equação para a dilatação do tempo deve valer caso o observador em movimento desloque-se com velocidade de módulo constante ao longo de um caminho poligonal, imaginando o movimento total como uma composição de MRUs. Se essa hipótese é válida, ele conclui, a equação da dilatação do tempo deve ser válida, também, para o movimento ao longo de um caminho curvo qualquer, imaginando o movimento total como uma composição de infinitos MRUs de deslocamento infinitesimal, todos com o mesmo valor de velocidade.

A suposição que Einstein fez constitui o que passou a ser chamado de hipótese do relógio [10. Esta generaliza a equação da dilatação do tempo para

$$
\frac{d t}{d \bar{\tau}}=\frac{1}{\sqrt{1-v^{2}(t) / c^{2}}},
$$

a qual relaciona o intervalo infinitesimal de tempo $d t$, registrado utilizando-se dois relógios sincronizados de um referencial inercial $\mathcal{S}$, com o intervalo infinitesimal de tempo próprio $d \bar{\tau}$, registrado por um único relógio do referencial $\overline{\mathcal{S}}$ em movimento qualquer. A hipótese do relógio, então, diz que, em cada instante, a dilatação do tempo é afetada somente pelo módulo da velocidade instantânea relativa entre estes referenciais, independendo de qualquer detalhe da aceleração do referencial $\overline{\mathcal{S}}[16,9$ De modo mais abrangente, pode-se encarar a hipótese do relógio como um terceiro postulado fundamental da TRE, o qual estende sua aplicação à descrição de movimentos acelerados.

$\mathrm{Na}$ Sec. 2.1, a definição do relógio-padrão inclui sua concordância com a hipótese do relógio. Naturalmente, isso só faz sentido se tal hipótese for realista. Este, de fato, é o caso e o seu acordo com o experimento foi verificado para acelerações transversas ao movimento da ordem de $10^{19} \mathrm{~m} / \mathrm{s}^{2}$ ao comparar-se o tempo de vida entre múons em repouso e múons em movimento circular 18, e para acelerações longitudinais da ordem de $10^{16} \mathrm{~m} / \mathrm{s}^{2}$ para bárions sigma freados em câmaras de bolhas de hidrogênio 19 .

Uma consequência da hipótese do relógio é a geometrização do espaçotempo. Para entender isso, vamos começar considerando uma partícula em MRU, cuja trajetória no espaçotempo está ilustrada pela reta em azul na Fig. 2a. Os dois postulados da TRE implicam que medições de deslocamento $(\Delta x)$ e intervalo de tempo $(\Delta t)$ para o movimento da partícula se relacionam da mesma forma em todos referenciais inerciais por meio da grandeza $\Delta s$, chamada intervalo espaçotemporal invariante [20]. Esta é definida por

$$
\Delta s^{2} \equiv-c^{2} \Delta t^{2}+\Delta x^{2}=-c^{2} \Delta t^{\prime 2}+\Delta x^{\prime 2},
$$

${ }^{9}$ Apesar da aceleração ter seu papel alterando a velocidade instantânea de $\overline{\mathcal{S}}$, o que a hipótese do relógio diz é que, independente de como esta velocidade muda, em cada instante a razão $d t / d \bar{\tau}$ só depende de $v(t)$. Uma analogia iluminadora é feita com a sensação térmica em um dia com vento frio 17: em cada momento, a sensação térmica de frio maior ou menor depende da velocidade do vento que te atinge naquele momento, mas não depende de como essa velocidade muda com o tempo.

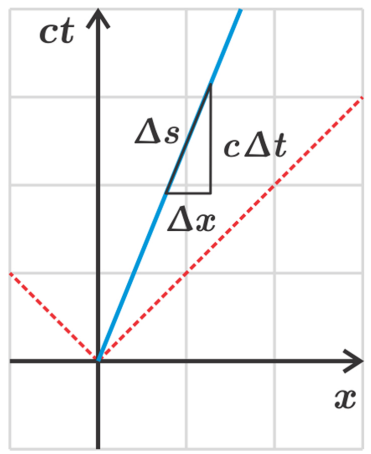

(a)

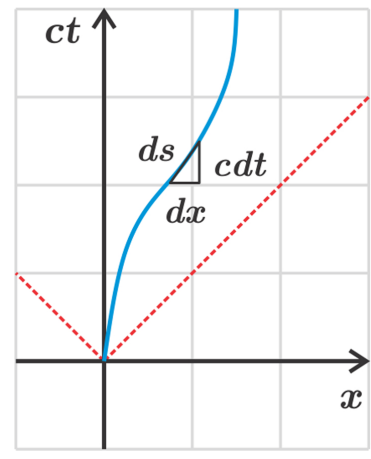

(b)
Figura 2

onde a segunda igualdade nos diz que outros referenciais inerciais medem outros valores para o deslocamento e o intervalo de tempo, mas que todos concordam sobre a relação entre tais medidas. Interpretando $\Delta s$ como uma medida da "distância espaçotemporal" entre dois pontos no espaçotempo, vemos que esta sugere que a partícula se desloca em um espaçotempo cuja geometria é hiperbólica. Para irmos além desta sugestão, é necessário analisar movimentos mais gerais em trechos infinitesimais de espaçotempo. Para isso, a hipótese do relógio é fundamental.

Primeiramente, vamos relacionar $\Delta s$ com o intervalo de tempo próprio $\Delta \tau^{\prime}$ medido em um referencial $\mathcal{S}^{\prime}$ que move-se junto a uma partícula em MRU. Neste, temse $\Delta t^{\prime}=\Delta \tau^{\prime}$ e $\Delta x^{\prime}=0$. Logo, da relação (8), segue que $\Delta s^{2}=-c^{2} \Delta t^{2}+\Delta x^{2}=-c^{2} \Delta \tau^{\prime 2}$. Sendo a velocidade da partícula $v=\Delta x / \Delta t$, temos também que $-c^{2} \Delta t^{2}+\Delta x^{2}=-c^{2} \Delta t^{2} / \gamma^{2}$. Finalmente, chegamos na relação $\Delta s^{2}=-c^{2} \Delta t^{2} / \gamma^{2}=-c^{2} \Delta \tau^{\prime 2}$ para uma partícula em MRU. Note que a última igualdade coincide com a dilatação do tempo (5).

O que a hipótese do relógio (7) nos diz é que podemos analisar a dilatação do tempo entre um referencial inercial $\mathcal{S}$ e um referencial não-inercial $\overline{\mathcal{S}}$ considerando o movimento do último como uma série de MRUs de deslocamentos infinitesimais 21. Isso permite fazer a generalização $d s^{2}=-c^{2} d t^{2} / \gamma^{2}=-c^{2} d \bar{\tau}^{2}$. Esta fornece o intervalo invariante infinitesimal $d s$ para uma partícula em um movimento qualquer (linha em azul na Fig. 2b), onde $d \bar{\tau}$ é o tempo próprio medido no referencial $\overline{\mathcal{S}}$ que se move junto à partícula $\sqrt{10}$ Como $-c^{2} d t^{2} / \gamma^{2}=-c^{2} d t^{2}+d x^{2}$, concluímos que, segundo um observador do referencial inercial $\mathcal{S}$, a trajetória da partícula é caracterizada por

$$
d s^{2}=-c^{2} d t^{2}+d x^{2}
$$

Como todos referenciais inerciais são equivalentes, em todos esta quantidade é calculada de modo análogo -

\footnotetext{
${ }^{10}$ Incidentalmente, note que todos referenciais, inerciais ou não, medem o mesmo valor de $d s$. Isso vem de $d s^{2}=-c^{2} d \bar{\tau}^{2}$, pois todos referenciais concordam quanto ao valor de $d \bar{\tau}$, já que este é medido usando-se somente um relógio.
} 
por exemplo, é calculada segundo $d s^{2}=-c^{2} d t^{\prime 2}+d x^{\prime 2}$ em um outro referencial inercial $\mathcal{S}^{\prime}$. Portanto, mesmo que o movimento de uma partícula seja governado por uma força resultante, cada trecho infinitesimal deste segue uma geodésica em um espaçotempo genuinamente hiperbólico: o chamado espaçotempo de Minkowski [22]. A hipótese do relógio, como vemos, é fundamental para a geometrização do espaçotempo e é, também, uma hipótese básica na construção da teoria da relatividade geral $[23]$.

\subsection{Dilatação do tempo para movimentos acelerados}

Considere a situação do problema dos gêmeos, onde Bob parte em viagem pelo espaço e depois retorna para reencontrar Ana. Tomemos dois eventos: o primeiro sendo o momento inicial com eles juntos e o segundo sendo o reencontro. Qual a relação entre o intervalo de tempo entre estes dois eventos como registrado por Ana e por Bob? Seja Ana um observador do referencial inercial $\mathcal{S}$ e Bob um do referencial acelerado $\overline{\mathcal{S}}$. De imediato, a hipótese do relógio (7) fornece a expressão para a dilatação do tempo entre os dois eventos 11

$$
\Delta \bar{\tau}=\int_{t_{1}}^{t_{2}} \sqrt{1-\frac{v^{2}(t)}{c^{2}}} d t .
$$

Como os dois eventos ocorrem em um mesmo local tanto para Ana quanto para Bob, só um relógio de cada referencial (o de Ana e o de Bob) são usados para registrar o intervalo de tempo até o reencontro. Ana registra $\Delta \tau=t_{2}-t_{1}$ e prevê que Bob registra $\Delta \bar{\tau}$ como dado por 10. . Como $\sqrt{1-v^{2}(t) / c^{2}} \leq 1$, segue que $\Delta \bar{\tau} \leq \Delta \tau$. Portanto, no momento do reencontro, Ana prevê que Bob terá envelhecido menos do que ela. Apesar de ser uma previsão, a conclusão de Ana deve ser comum a todos referenciais, inclusive ao de Bob, pois ela e Bob medem o tempo até o reencontro com somente um relógio de seus referenciais.

A pergunta, agora, não é "qual é a previsão de Bob?" pois sabemos qual ela deve ser, mas, sim, "como Bob faz sua previsão?" Ao contrário do que uma intuição pouco cuidadosa poderia sugerir, temos que

$$
\Delta \tau \neq \int_{\bar{t}_{1}}^{\bar{t}_{2}} \sqrt{1-\frac{\bar{v}^{2}(\bar{t})}{c^{2}}} d \bar{t} .
$$

Se esta equação envolvesse uma igualdade, Bob chegaria a uma conclusão contrária à de Ana, caracterizando um paradoxo. Naturalmente, não há paradoxos na natureza, o que fornece uma justificativa lógica para o símbolo de desigualdade. A justificativa formal vem de

$$
\frac{d \bar{t}}{d \tau} \neq \frac{1}{\sqrt{1-\bar{v}^{2} / c^{2}}} .
$$

\footnotetext{
${ }^{11} \mathrm{O}$ resultado da integral em 10 exige conhecimento da velocidade instantânea $v(t)$ do referencial acelerado $\overline{\mathcal{S}}$ e será obtida para o caso do movimento sob ação de uma força constante na Sec. 3
}

Como (11) claramente vem desta relação, o motivo do símbolo de desigualdade continua sem ser respondido. Será? Esta relação ressalta o que a hipótese do relógio $n \tilde{a} o$ diz. A hipótese do relógio, $d t / d \bar{\tau}=\gamma$, é uma relação entre o intervalo $d t$ medido em um referencial inercial e o intervalo próprio $d \bar{\tau}$ medido em um referencial qualquer. Portanto, qualquer previsão sobre $d \bar{t} / d \tau$ está fora do escopo da hipótese do relógio, pois envolve o intervalo $d \bar{t}$ medido em um referencial qualquer, mas não necessariamente inercial. Logo, a hipótese do relógio não pode ser usada como ponto de partida para calcular-se $\Delta \tau$, o que justifica (11) e evita conclusões paradoxais.

As implicações de 12 são profundas e dizem respeito às conclusões que observadores de diferentes referenciais fazem a respeito da geometria do espaçotempo. Como discutido no final da seção anterior, observadores de um referencial inercial $\mathcal{S}$ medem o intervalo invariante entre dois eventos próximos como sendo $d s^{2}=-c^{2} d t^{2}+d x^{2}$. Para essa conclusão, a hipótese do relógio foi fundamental, e a consequência é que tais observadores constatam que o espaçotempo possui geometria hiperbólica. Por outro lado, por causa de $(12)$, medidas para esse mesmo par de eventos feitas por um observador do referencial não-inercial $\overline{\mathcal{S}}$ fornecem $d s^{2} \neq-c^{2} d \bar{t}^{2}+d \bar{x}^{2}$. Isso significa que ele observa o espaçotempo como se este possuísse uma geometria diferente da hiperbólica. Esse é um efeito do movimento acelerado do observador, análogo ao surgimento de forças fictícias em um referencial não-inercial, e assim que um estado inercial é atingido, o observador volta a experimentar o espaçotempo hiperbólico descrito por (9). No contexto do problema dos gêmeos, as consequências do movimento não-inercial sobre o que é observado por Bob serão exploradas na Sec. 4.3 .

As conclusões desta seção são gerais e resultados particulares ao problema dos gêmeos dependem dos detalhes do movimento de Bob. Nas próximas seções deste artigo, analisaremos o problema do ponto de vista de ambos os gêmeos considerando Bob em movimento retilíneo uniformemente variado (MRUV).

\section{O Problema dos Gêmeos do Ponto de Vista de Ana}

Tendo em vista uma resolução do problema dos gêmeos suficientemente simples, mas sem perda de detalhes importantes, consideraremos apenas movimentos confinados ao eixo $x$, como especificado ao fim da Sec. 2.2. Ana será o gêmeo em repouso no referencial inercial $\mathcal{S}$ e Bob o gêmeo em repouso no referencial acelerado $\overline{\mathcal{S}}$.

Em uma situação mais realista, Bob estaria inicialmente em repouso junto a Ana. Ele, então, se afastaria dela até parar, daria meia-volta e retornaria até terminar o movimento em repouso junto a ela novamente. Esta formulação do problema foi considerada em [24]. Aqui, vamos considerar um movimento mais simples para Bob, o que trará a vantagem de uma simplicidade matemática e clareza conceitual maior. 
Consideraremos que Bob está, a todo instante, sob ação de uma força constante e paralela ao seu movimento. Nesta situação, assumiremos que Bob encontra Ana pela primeira vez ao passar por ela em um movimento desacelerado. A partir de então, ele afasta-se até parar e retorna de modo acelerado para o segundo encontro com ela. O problema, portanto, é determinar o tempo transcorrido entre os dois encontros, tanto do ponto de vista de Ana quanto do de Bob. O que mostraremos é que ambos concordam sobre o registro que cada um faz para este tempo e que, no reencontro, a conclusão é que Bob envelheceu menos que Ana.

O que se segue nesta seção é dedicado à análise do problema do ponto de vista de Ana.

\subsection{Preliminares}

No âmbito da TRE, qualquer que seja o movimento de Bob, em qualquer instante $t^{*}$ sempre é possível utilizar as transformações de Lorentz para encontrar um referencial inercial $\mathcal{S}^{\prime}$ momentaneamente em repouso relativo a ele, isto é, que o observa com velocidade relativa $\boldsymbol{u}$ nula, $\boldsymbol{u}\left(t^{*}\right)=0$. O mesmo não é verdade para a aceleração $\boldsymbol{a}$ de Bob. Se, em um dado instante $t^{\star}$, um referencial inercial observa que $\boldsymbol{a}\left(t^{\star}\right) \neq 0$, todos outros referenciais inerciais concordam com isso neste instante. Isso decorre da invariância da grandeza escalar $\alpha$, chamada aceleração própria, definida por $\alpha^{2} \equiv a^{2} /\left(1-u^{2} / c^{2}\right)^{3}$ no caso do movimento unidimensional 20$]{ }^{12}$ Como $1-u^{2} / c^{2} \neq 0$, se $a\left(t^{\star}\right) \neq 0$ para um referencial inercial, também o será para qualquer outro. Na TRE, enquanto, em cada instante, o estado de repouso ou movimento é relativo, o "estado de aceleração" é absoluto.

Como a aceleração própria $\alpha$ é invariante, mas o mesmo não pode ser dito da aceleração "ordinária" $\boldsymbol{a}$, devemos ser cautelosos ao usar o termo MRUV no contexto da TRE. Intuitivamente, vamos definir que tal movimento é aquele fruto da ação de uma força $\boldsymbol{F}$ constante. A versão relativística da segunda lei de Newton, $\boldsymbol{F}=d \boldsymbol{p} / d t$, sendo o momento relativístico $\boldsymbol{p} \equiv m \boldsymbol{u} / \sqrt{1-u^{2} / c^{2}}$, não se reduz ao formato simples $\boldsymbol{F}=m \boldsymbol{a}$ devido ao fator extra no denominador de $\boldsymbol{p}$, mas, no caso particular do movimento retilíneo com $\boldsymbol{F}$ paralelo à $\boldsymbol{u}$, ela se reduz a $F=m a /\left(1-u^{2} / c^{2}\right)^{3 / 2}=m \alpha$. Portanto, o que definimos como MRUV na TRE corresponde ao movimento retilíneo com aceleração própria constante.

\subsection{Cinemática de Bob segundo Ana}

A descrição do movimento de Bob será feita segundo o referencial inercial $\mathcal{S}$, no qual Ana está em repouso na posição $X_{\mathrm{A}}$. Segundo a definição de referencial feita na

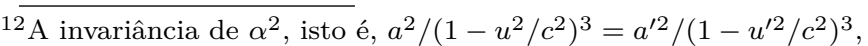
pode ser verificada relacionando-se $u$ e $u^{\prime}$ por meio da regra de Einstein para a adição de velocidades, $u=\left(u^{\prime}+v\right) /\left(1+u^{\prime} v / c^{2}\right)$, sendo $v$ a velocidade entre $\mathcal{S}$ e $\mathcal{S}^{\prime}$, e usando-se esta para calcular $a=d u / d t$ por meio da relação $d t=\gamma\left(1+u^{\prime} v / c^{2}\right) d t^{\prime}$ obtida das transformações de Lorentz 3 .
}

Sec. 2.1. podemos considerar Ana como sendo um dos observadores que constituem este referencial. Em particular, o tempo registrado pela coordenada $t$ do referencial $\mathcal{S}$ sempre coincide com o tempo registrado pelo relógio de Ana.

Bob está em um referencial cujo movimento é causado unicamente pela ação de uma força $\boldsymbol{F}$ constante ao longo da direção do seu movimento retilíneo. Modelaremos a situação da seguinte maneira: a força $\boldsymbol{F}$ promove uma aceleração própria positiva em Bob $(\alpha>0)$ e seu movimento é tal que ele encontra-se em repouso em $x=c^{2} / \alpha$ no instante $t=0{ }^{13}$ A posição de Bob, $x(t)$, e sua velocidade, $u(t)$, são obtidas por integrações no tempo de $F=m a /\left(1-u^{2} / c^{2}\right)^{3 / 2}=m \alpha$. Com as considerações que fizemos, estas são dadas por

$$
x^{2}-c^{2} t^{2}=\left(\frac{c^{2}}{\alpha}\right)^{2}, \quad u(t)=\frac{\alpha t}{\sqrt{1+(\alpha t / c)^{2}}} .
$$

A primeira expressão nos diz que o movimento de Bob descreve uma hipérbole no espaçotempo, e não uma parábola como no caso não-relativístico. Em consonância a isso, da expressão de $u(t)$, vemos que sua velocidade não cresce indefinidamente, pois tem $c$ como limite superior. É interessante notar que, ainda que Bob jamais movase tão rápido quanto a luz, seu movimento acelerado torna possível que, em certas situações, ele escape de um raio luminoso projetado em sua direção. Em particular, nenhum raio luminoso disparado em $t \geq 0$ a partir de $x<0$ jamais chegará a Bob. Esta conclusão está ilustrada no diagrama de espaçotempo da Fig. 3, o qual contém as trajetórias de Ana, Bob e um raio luminoso sob as condições mencionadas. Ainda que essa peculiaridade diga respeito ao que Bob é capaz de ver, elas sugerem que Bob não é capaz de observar ${ }^{14}$ eventos em certos pontos do espaçotempo. Exploraremos o que Bob observa em maiores detalhes na Sec. 4.3 .

Da Fig. 3. fica claro que Ana e Bob se encontram duas vezes somente se a posição de Ana, $X_{A}$, for maior que a posição de Bob em $t=0, x(0)=c^{2} / \alpha$. Por isso, definiremos a posição de Ana como $X_{\mathrm{A}} \equiv k\left(c^{2} / \alpha\right)$, onde $k$ é um número real maior do que 1 .

Consideremos, agora, os registros de tempo de Ana e Bob. Quando Ana observa Bob ir de uma posição $\left(x_{1}\right)$ a outra $\left(x_{2}\right)$, dois relógios do referencial $\mathcal{S}$ (um em cada um destes locais) são utilizados para registrar os instantes $t_{1}$ e $t_{2}$, respectivamente, no qual Bob chega a estas posições. Por outro lado, Bob julga estar sempre em repouso na mesma posição em seu referencial $\overline{\mathcal{S}}$, logo usa somente um relógio (o seu) para registrar o intervalo de tempo próprio $\Delta \bar{\tau}$. Como esses registros se relacionam? A resposta vem da hipótese do relógio discutida na Sec. 2.4. Esta conduz à expressão 10, , onde $v(t)$ é a velocidade do referencial

\footnotetext{
${ }^{13}$ Não há nada de especial na escolha de Bob em $x(0)=c^{2} / \alpha$, mas esta deixará as expressões que obteremos com uma aparência mais simples.

${ }^{14}$ Ver nota de rodapé 5
} 


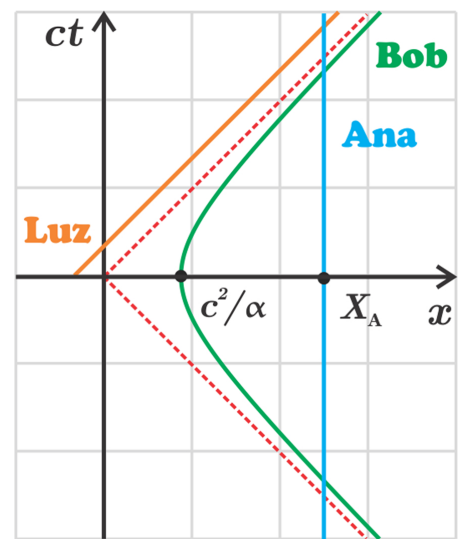

Figura 3

$\overline{\mathcal{S}}$, que se move junto à Bob, dada pela segunda expressão de (13). Com isso, encontramos

$$
\begin{aligned}
\Delta \bar{\tau} & =\int_{t_{1}}^{t_{2}} \frac{d t}{\sqrt{1+(\alpha t / c)^{2}}} \\
& =\frac{c}{\alpha}\left[\operatorname{senh}^{-1}\left(\frac{\alpha t_{2}}{c}\right)-\operatorname{senh}^{-1}\left(\frac{\alpha t_{1}}{c}\right)\right] .
\end{aligned}
$$

Esta é a equação para a dilatação do tempo, como observada por Ana, para o movimento de Bob entre dois pontos quaisquer. Para deixá-la em um formato mais simples, por conveniência, vamos considerar que tanto os relógios do referencial $\mathcal{S}$ quanto o de Bob foram ajustados para marcar $t=\bar{\tau}=0$ quando Bob passa em $x=c^{2} / \alpha$, isto é, quando Ana e Bob estão em repouso relativo. Com essa escolha, os tempos medidos em relação ao instante zero se relacionam por

$$
t(\bar{\tau})=\frac{c}{\alpha} \operatorname{senh}\left(\frac{\alpha \bar{\tau}}{c}\right) .
$$

Como o comportamento da função seno hiperbólico implica em $t \geq \bar{\tau} 15$ Ana afirma que o tempo transcorre mais lentamente para Bob do que para ela. Vimos na Sec. 2.3 que essa é uma conclusão particular ao referencial de Ana, pois observadores de outros referenciais, como Bob, geralmente discordam alegando que Ana chega a tal conclusão comparando dois relógios fora de sincronia com o relógio de Bob. Para evitar tal ambiguidade, na próxima seção, compararemos o registro feito por Ana e Bob para o intervalo entre seus dois encontros, pois, para tal registro, cada um usa somente um relógio.

Futuramente, na Sec. 4.1. também precisaremos da relação entre $x$ e $\bar{\tau}$. Esta é obtida usando-se (15) para eliminar $t$ na expressão para a posição de Bob, dada em 13. O resultado é

$$
x(\bar{\tau})=\frac{c^{2}}{\alpha} \cosh \left(\frac{\alpha \bar{\tau}}{c}\right) .
$$

\footnotetext{
${ }^{15}$ Além do método gráfico, uma maneira prática de constatar isso é pela expansão em série de Taylor do seno hiperbólico: $\operatorname{senh} x=$ $x+x^{3} / 3$ ! $+x^{5} / 5 !+\cdots>x$ para todo $x>0$.
}

Imaginando a trajetória de Bob no espaçotempo como parametrizada pelo seu tempo próprio $\bar{\tau}$, podemos encarar as expressões 15 e 16 como as equações paramétricas da sua trajetória.

\subsection{Conclusões de Ana}

Para uma comparação entre os tempos registrados por Ana e Bob a respeito da qual todos referenciais concordem, consideraremos o intervalo entre seus dois encontros no ponto $x=X_{\mathrm{A}}$. Pela simetria do movimento de Bob, digamos que o primeiro encontro ocorre no instante $-T$ e o segundo no instante $T$ no relógio de Ana. Assim, ela registra o intervalo de tempo próprio $\Delta \tau=2 T$ e, da relação 14, prevê que Bob registra $\Delta \bar{\tau}=(2 c / \alpha) \operatorname{senh}^{-1}(\alpha T / c)$. Da razão

$$
\frac{\Delta \tau}{\Delta \bar{\tau}}=\frac{(\alpha T / c)}{\operatorname{senh}^{-1}(\alpha T / c)} \geq 1,
$$

e em comparação ao primeiro encontro com Bob, Ana conclui que, no momento do segundo encontro, terá envelhecido mais do que ele pelo fator de $\Delta \tau / \Delta \bar{\tau}{ }^{16}$

Poderíamos ter a falsa impressão de que a dilatação do tempo (17) depende da aceleração de Bob. No entanto, considerando a hipótese do relógio, discutida na Sec. 2.4. sabemos que este não deve ser o caso. $\mathrm{O}$ que faremos a seguir é mostrar que a razão $\Delta \tau / \Delta \bar{\tau}$ só depende do módulo da velocidade relativa entre Ana e Bob em seus encontros.

O instante $T$ e o módulo da velocidade de Bob nos dois encontros, $U \equiv|u(T)|$, podem ser obtidos de (13) tomando-se $x=X_{\mathrm{A}} \equiv k c^{2} / \alpha$. O resultado é

$$
\begin{gathered}
T=\frac{c}{\alpha} \sqrt{k^{2}-1}=\frac{c}{\alpha} \operatorname{senh} \phi, \\
\frac{U}{c}=\frac{\sqrt{k^{2}-1}}{k} \equiv \tanh \phi .
\end{gathered}
$$

A segunda igualdade de 19 nada mais é do que a definição da rapidez $\phi$ de Bob calculada no instante $T{ }^{17} \mathrm{Tal}$ definição permite identificar $\phi$ como sendo o ângulo do triângulo hiperbólico da Fig. 4a, o que justifica a segunda igualdade de (18). Por conveniência futura, também podemos identificá-lo como o ângulo na Fig. 4b.

Como discutido no início desta seção, Ana registra o intervalo $\Delta \tau=2 T$ entre os dois encontros e prevê que Bob registra $\Delta \bar{\tau}=(2 c / \alpha) \operatorname{senh}^{-1}(\alpha T / c)$. Finalmente, utilizando (18) para eliminar $T$, o intervalo registrado por Ana é

$$
\Delta \tau=2 T=\frac{2 c}{\alpha} \operatorname{senh} \phi=\frac{2 \gamma_{U} U}{\alpha},
$$

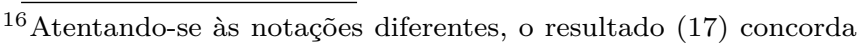
por exemplo, com o obtido na Eq. (9.6) de 6] e o resultado da Sec. $\mathrm{B}$ de 7 .

${ }^{17} \mathrm{Na}$ TRE, a rapidez $\phi$ de uma partícula é definida por $\tanh \phi(t) \equiv$ $u(t) / c 22$. Esta pode ser interpretada como uma definição mais natural de "velocidade" para o espaçotempo da TRE, pois a rapidez relativa é obtida por uma regra simples de adição $\phi_{A C}=\phi_{A B}+$ $\phi_{B C}$.
} 
(a)

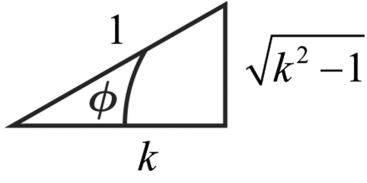

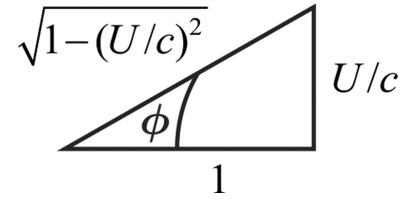

(b)
Figura 4

sendo o valor de $\sinh \phi$ obtido da Fig. $4 \mathrm{~b}$, onde $\gamma_{U} \equiv$ $1 / \sqrt{1-U^{2} / c^{2}}$ é o fator de Lorentz entre Ana e Bob nos instantes $\pm T$. Para o registro previsto para Bob, tem-se

$$
\Delta \bar{\tau}=\frac{2 c}{\alpha} \operatorname{senh}^{-1}\left(\frac{\alpha T}{c}\right)=\frac{2 c \phi}{\alpha}=\frac{2 c}{\alpha} \tanh ^{-1}\left(\frac{U}{c}\right),
$$

onde, novamente, usamos a Fig. 4b para eliminar $\phi$. A razão entre os intervalos é

$$
\frac{\Delta \tau}{\Delta \bar{\tau}}=\frac{\gamma_{U} U / c}{\tanh ^{-1}(U / c)} \geq 1
$$

O comportamento desta razão em função de $U$ está representado no gráfico da Fig. 5 o qual ilustra a conclusão de que $\Delta \tau \geq \Delta \bar{\tau}$. Em particular, vemos que $\Delta \tau / \Delta \bar{\tau}$ independe da aceleração de Bob, como esperado tendo-se em mente a hipótese do relógio, e é função somente da velocidade relativa $U$ entre Ana e Bob no momento dos encontros.

\section{O Problema dos Gêmeos do Ponto de Vista de Bob}

Por vir de uma comparação entre tempos próprios, todos referenciais inerciais, inclusive um instantaneamente em repouso junto a Bob, devem chegar às mesmas conclusões que Ana, discutidas na Sec. 3.3. Portanto, Bob deve constatar que seu relógio registra (21) para o intervalo

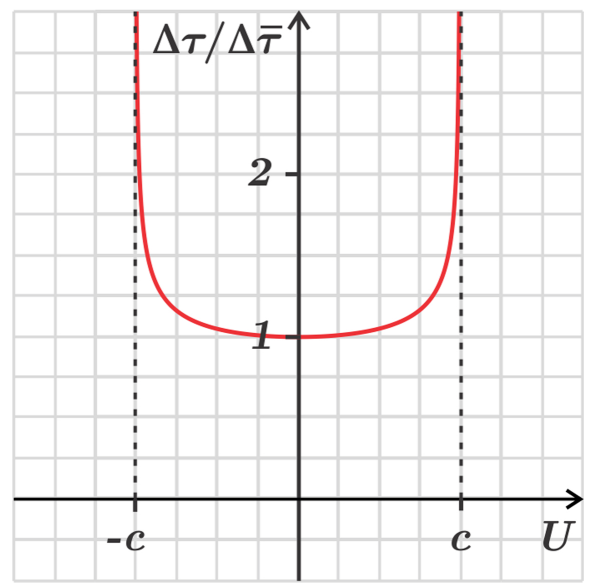

Figura 5 entre seus dois encontros com Ana e também prever que ela registra 20 para tal intervalo. Para chegar a tal resultado, é necessário entender como Bob faz medidas de distância e registros de tempo. Portanto, primeiro começamos construindo o referencial $\overline{\mathcal{S}}$ de Bob.

\subsection{Construção do referencial de Bob}

Seguindo a definição de referencial feita na Seção 2.1. na construção do referencial uniformemente acelerado $\mathcal{S}$, no qual Bob está em repouso, consideramos Bob como sendo apenas um dos infinitos observadores que constituem tal referencial. A partir do conjunto de réguas e relógios-padrão carregados por tais observadores, podemos localizar eventos no espaçotempo por meio de coordenadas $(\bar{x}, \bar{t})$ deste referencial. Para que $\overline{\mathcal{S}}$ seja um referencial rígido, seus observadores devem estar em repouso relativo um ao outro, de modo que a separação $\delta \bar{x}$ entre observadores adjacentes nunca mude. Do ponto de vista do referencial inercial $\mathcal{S}$, a separação $\delta x$ entre estes observadores adjacentes deve satisfazer, em cada instante, a contração do comprimento prevista pela TRE, $\delta x=\delta \bar{x} / \gamma$, sendo $\gamma=\gamma(t)$ o fator de Lorentz instantâneo entre o par de observadores adjacentes de $\overline{\mathcal{S}}$ e o referencial $\mathcal{S}[25,26,18$

Exigir que o referencial $\overline{\mathcal{S}}$ seja rígido implica que os observadores que o compõem não podem possuir a mesma aceleração própria. Para entender isso, imagine se este não fosse o caso. Considere um par de observadores, um satisfazendo $x_{1}(0)=c^{2} / \alpha$ e o outro $x_{2}(0)=c^{2} / \alpha+\delta x$, sendo $\delta x$ o comprimento da régua que mede a separação entre os dois no referencial $\mathcal{S}$. Se ambos possuem a mesma aceleração própria $\alpha$, a trajetória do primeiro observador é descrita pela hipérbole $x^{2}-c^{2} t^{2}=c^{4} / \alpha^{2}$ e a do segundo por $(x-\delta x)^{2}-c^{2} t^{2}=c^{4} / \alpha^{2}$. Isso significa que, do ponto de vista de $\mathcal{S}$, o comprimento da régua entre os dois é $\delta x$ em todos instantes, independente de sua velocidade. A situação está ilustrada na Fig. 6, onde as hipérboles em verde representam os observadores e as retas em marrom representam a régua entre eles em diferentes instantes. Como a contração do espaço prevê que $\delta x=\delta \bar{x} / \gamma$, sendo $\gamma=\gamma(t)$, estes dois observadores não podem estar em repouso relativo do ponto de vista do referencial $\overline{\mathcal{S}}$. Pelo contrário, para $t>0$, por exemplo, eles devem estar afastando-se um do outro, tensionando a régua conforme $\delta \bar{x}$ aumenta, de modo a manter $\delta x$ inalterado. Portanto, um referencial uniformemente acelerado não pode ser rígido caso todos observadores que o compõem, junto às

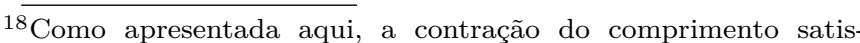
faz a hipótese do comprimento 10$]$. Esta diz que $\delta \bar{x} / \delta x=$ $1 / \sqrt{1-v^{2}(t) / c^{2}}$, onde $\delta \bar{x}$ é o comprimento infinitesimal de uma régua no seu referencial de repouso $\overline{\mathcal{S}}$ e $\delta x$ é o seu comprimento como observado em $\mathcal{S}$. Segundo esta hipótese, a contração do comprimento depende apenas do módulo da velocidade instantânea relativa entre $\mathcal{S}$ e $\overline{\mathcal{S}}$.
} 


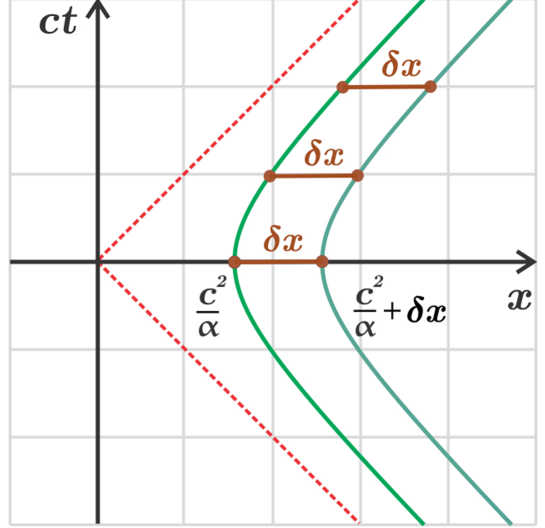

Figura 6

suas réguas-padrão, movam-se com a mesma aceleração própria 19

Considerando que cada observador de $\overline{\mathcal{S}}$ move-se, do ponto de vista de $\mathcal{S}$, com um valor específico $\alpha_{\text {obs }}$ de aceleração própria, escolheremos condições iniciais análogas às de Bob para o movimento de cada um: o observador em $\bar{x}$ de $\overline{\mathcal{S}}$ satisfaz $x(0)=c^{2} / \alpha_{\text {obs }}$ e $u(0)=0$. Isso garante que Bob mantém-se sempre em repouso relativo ao referencial $\overline{\mathcal{S}}$. De modo análogo à Bob, a trajetória em $\mathcal{S}$ de cada observador de $\overline{\mathcal{S}}$ será descrita pela hipérbole $x^{2}-c^{2} t^{2}=\left(c^{2} / \alpha_{\text {obs }}\right)^{2}$. Esta equação junto à condição de rigidez $(\delta x=\delta \bar{x} / \gamma)$ determina a aceleração própria de cada observador em função de $\bar{x}$ [29]. Em particular, encontramos $\alpha_{\text {obs }}(\bar{x})=c^{2} / \bar{x}{ }^{20}$ Este resultado carrega a escolha de que a origem do referencial $\mathcal{S}$ coincide com a origem do referencial $\overline{\mathcal{S}}$ no instante $t=0$ e também que o observador em $\bar{x}=c^{2} / \alpha$ possui aceleração própria $\alpha$, identificando-o como sendo Bob.

Uma escolha conveniente é ajustar a coordenada temporal $\bar{t}$ do referencial $\overline{\mathcal{S}}$ para sempre coincidir com o tempo próprio registrado pelo relógio de Bob. Para isso, a definimos como $\bar{t} \equiv c^{2} \bar{\tau}_{\text {obs }} / \alpha \bar{x}$. Nesta relação, ressaltamos que o tempo próprio de cada observador, $\bar{\tau}_{\text {obs }}$, é uma função de $\bar{x}$. Essa dependência é esperada, pois cada observador de $\overline{\mathcal{S}}$ possui uma aceleração própria diferente, de modo que, segundo o referencial $\mathcal{S}$, seus relógios exibem gradual perda de sincronia devido à dilatação do tempo causada por suas velocidades relativas. Da definição de $\bar{t}$, o relógio de cada observador de $\overline{\mathcal{S}}$ está ajustado para marcar $\bar{\tau}_{\text {obs }}=0$ em $\bar{t}=0$. Como, na Sec. 3.2 ajustamos

\footnotetext{
${ }^{19}$ Essa é uma das sutilezas que residem por trás do chamado "paradoxo das espaçonaves de Bell", originalmente publicado em [27] e popularizado por John S. Bell em [28].

${ }^{20}$ Para deduzir isso, considere dois observadores de $\overline{\mathcal{S}}$ com acelerações próprias diferindo de $\delta \alpha_{\text {obs }}$. A separação $\delta x$ entre os dois em um instante $t$ é obtida variando-se $x$ em $x^{2}-c^{2} t^{2}=\left(c^{2} / \alpha_{\text {obs }}\right)^{2}$, resultando em $\delta x=-\left(c^{4} / \alpha_{\text {obs }}^{3} x\right) \delta \alpha_{\text {obs }}$. O fator $\gamma$ entre $\mathcal{S}$ e $\overline{\mathcal{S}}$ neste instante vale $\alpha_{\text {obs }} x / c^{2}$, obtido utilizando-se a velocidade da Eq. 13. com $\alpha_{\text {obs }}$ no lugar de $\alpha$. Com estes resultados e a condição $\delta x=\delta \bar{x} / \gamma$, uma integração simples resulta em $\alpha_{\text {obs }}(\bar{x})=c^{2} / \bar{x}$ ao escolher-se $\alpha_{\text {obs }}=\alpha$ em $\bar{x}=c^{2} / \alpha$.
}

o relógio de Bob para indicar $\bar{\tau}=0$ em $t=0$, neste mesmo instante também temos $\bar{t}=0$ no referencial $\overline{\mathcal{S}}$.

A cinemática de cada observador de $\overline{\mathcal{S}}$ é análoga a de Bob, resumida pelas equações $(13), 15$ e 116 da Sec. 3.2 mas com aceleração própria dada por $\alpha_{\text {obs }}(\bar{x})=c^{2} / \bar{x}$ e tempo próprio dado por $\bar{\tau}_{\mathrm{obs}}=\alpha \bar{x} \bar{t} / c^{2}$. Primeiramente, segundo o referencial $\mathcal{S}$, a trajetória de cada um desses observadores é uma hipérbole no espaçotempo, $x^{2}-$ $c^{2} t^{2}=\bar{x}^{2}$. Cada uma destas hipérboles representa uma linha de $\bar{x}$ constante em $\overline{\mathcal{S}}$. Por fim, a razão entre a coordenada $t$ e a coordenada $x$ de um observador de $\overline{\mathcal{S}}$ fornece a relação $c t / x=\tanh \left(\alpha_{\text {obs }} \bar{\tau}_{\text {obs }} / c\right)$, que pode ser reescrita como $c t / x=\tanh (\alpha \bar{t} / c)$. Portanto, linhas de simultaneidade em $\overline{\mathcal{S}}$, isto é, linhas de $\bar{t}$ constante, são retas de inclinação $\tanh (\alpha \bar{t} / c)$ segundo o referencial $\mathcal{S}$. Desta análise, representamos o referencial $\overline{\mathcal{S}}$, como observado de $\mathcal{S}$ em $t=0$, na Fig. 7 .

Outros dois aspectos merecem destaque na Fig. 7. $\mathrm{O}$ primeiro é que, como $\alpha_{\text {obs }}>0$ para todos observadores de $\overline{\mathcal{S}}$, a relação $\alpha_{\text {obs }}(\bar{x})=c^{2} / \bar{x}$ nos diz que o sistema de coordenadas $(\bar{x}, \bar{t})$ restringe-se à região $\bar{x}>0$. O segundo é que nenhum destes observadores pode ocupar a posição $\bar{x}=0$. Da equação da trajetória de cada observador $\left(x^{2}-c^{2} t^{2}=\bar{x}^{2}\right)$, um observador em $\bar{x}=0$ deveria deslocar-se tão rápido quanto à luz, o que é impossível. Logo, $\bar{x}=0$ é um ponto singular no referencial $\overline{\mathcal{S}}$. Por isso, destacamos em vermelho a linha de $\bar{x}=0$ na Fig. 7. Consequências disso serão discutidas na Sec. 4.3

\subsection{Transformações de coordenada entre $\mathcal{S}$ e $\overline{\mathcal{S}}$}

Construído o referencial de Bob, o próximo passo é obter as transformações que relacionam as coordenadas do seu referencial às do referencial de Ana. Para isso, utilizamos o resultado $\alpha_{\text {obs }}=c^{2} / \bar{x}$ e a definição $\bar{t} \equiv c^{2} \bar{\tau}_{\text {obs }} / \alpha \bar{x}$ junto às versões de 15 e 16 para um observador de $\overline{\mathcal{S}}$ qualquer. O resultado é

$$
c t=\bar{x} \operatorname{senh}\left(\frac{\alpha \bar{t}}{c}\right), \quad x=\bar{x} \cosh \left(\frac{\alpha \bar{t}}{c}\right) .
$$

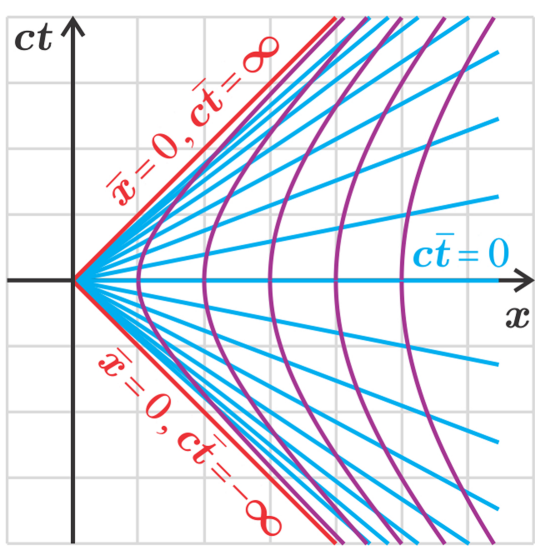

Figura 7 
Estas são as transformações que relacionam as coordenadas $(x, t)$ do referencial inercial $\mathcal{S}$ com as coordenadas $(\bar{x}, \bar{t})$ do referencial acelerado $\overline{\mathcal{S}}$, também chamadas de transformações de Rindler 30]. As transformações inversas são

$$
c \bar{t}=\frac{c^{2}}{\alpha} \tanh ^{-1}\left(\frac{c t}{x}\right), \quad \bar{x}=\sqrt{x^{2}-c^{2} t^{2}} .
$$

Enquanto as transformações de Lorentz (1) e suas inversas (3) são estruturalmente idênticas, o mesmo não pode ser dito a respeito das transformações de Rindler e suas inversas. Isso, claro, é fruto da não- equivalência entre os referenciais $\mathcal{S}$ e $\overline{\mathcal{S}}$. Em particular, uma consequência é que, enquanto eventos em qualquer ponto do espaçotempo são identificáveis pelas coordenadas $(x, t)$, somente aqueles dentro da região $0<x<\infty \mathrm{e}-x<c t<x$ podem ser identificados por coordenadas $(\bar{x}, \bar{t}){ }^{21}$ Fisicamente, isso significa que somente eventos nesta região podem ser observados pelos observadores de $\overline{\mathcal{S}}$.

\subsection{Espaçotempo observado por Bob}

Por causa da conclusão que encerra a seção anterior, Bob observa o espaçotempo de modo diferente do de Ana. Enquanto Ana observa que distâncias e intervalos de tempo infinitesimais se relacionam por $d s^{2}=-c^{2} d t^{2}+$ $d x^{2}$, Bob observa que estes se relacionam por ${ }^{22}$

$$
d s^{2}=-\left(\frac{\alpha \bar{x}}{c^{2}}\right)^{2} c^{2} d \bar{t}^{2}+d \bar{x}^{2} .
$$

Para Bob, é como se ele vivesse em um espaçotempo com características diferentes daquele onde Ana vive. Ambos estão no espaçotempo de Minkowski, caracterizado por (9). Apesar disso, somente a região $0<x<\infty$ e $-x<$ $c t<x$ deste espaçotempo pode ser observada por Bob 23 Por este motivo, suas medições de $d \bar{x}$ e $d \bar{t}$ equiparem-se às feitas em um espaçotempo caracterizado por (25), o chamado espaçotempo de Rindler.

Por causa do seu movimento acelerado, Bob observa a luz propagar-se com velocidade diferente de $c$ 31]. O motivo disso reside em como Bob observa o próprio espaçotempo. De acordo com Ana, o movimento da luz é tal que o deslocamento espacial $(d x)$ sempre coincide com o temporal $(c d t)$, pois $c=d x / d t$. Logo, o intervalo invariante (9) para o movimento da luz sempre se anula. Isso é verdade em qualquer referencial inercial e, em particular,

\footnotetext{
${ }^{21}$ Isso pode ser constatado pela Fig. 7 De modo mais formal, a primeira relação em 24 é válida para $-1<c t / x<1$, ou seja, para $-x<c t<x$. A mesma condição garante a validade da segunda relação. Por fim, tal condição é respeitada somente para $0<x<\infty$.

${ }^{22}$ Esta relação pode ser obtida calculando-se as diferenciais $d x$ e $d t$ em termos de $d \bar{x}$ e $d \bar{t}$ por meio de 23 e substituindo-as em $d s^{2}=-c^{2} d t^{2}+d x^{2}$.

${ }^{23}$ Cuidado com a diferença entre ver e observar (nota de rodapé 5. Bob pode até ver um evento fora da região $0<x<\infty$ e $-x<c t<x$, mas não pode observá-lo, pois, fora desta região, não há observadores de $\overline{\mathcal{S}}$ para registrar as coordenadas do evento.
}

$d s^{2}=0$ é verdade também em um referencial inercial que esteja em repouso instantâneo junto à Bob. Por observar um espaçotempo de Rindler, Bob calcula $d s^{2}$ segundo 25 e observa a luz propagar-se com velocidade $\bar{v}=d \bar{x} / d t= \pm \alpha \bar{x} / c$ que varia de acordo com a posição $\bar{x}$ da luz. Por exemplo, sinais luminosos propagando-se em direção a $\bar{x}=0$ movem-se de modo cada vez mais lento conforme aproximam-se do seu destino, nunca efetivamente chegando nele, como se ficassem "congelados" em suas proximidades. ${ }^{24}$ Apesar deste comportamento peculiar, o espaçotempo observado por Bob exibe curvatura nula 29], pois nada mais é do que o espaçotempo de Minkowski observado segundo um referencial acelerado - todas peculiaridades descritas sumiriam se, em algum momento, o movimento de Bob se tornasse inercial.

Na próxima seção, desejaremos ilustrar o movimento de Ana, como observado por Bob, em um diagrama de espaçotempo. Para interpretá-lo, ajudará saber qual é a equação da trajetória de raios luminosos no referencial $\overline{\mathcal{S}}$. Esta é obtida integrando-se a expressão para a velocidade da luz, sendo o resultado $\bar{t}= \pm(c / \alpha) \ln \left(\bar{x} / \bar{x}_{o}\right)$, onde $\bar{x}_{o}$ é a posição do raio luminoso no instante $\bar{t}=0$. Essa equação define o "cone" de luz no referencial de Bob. Por exemplo, neste referencial, o "cone" de luz de Bob no instante $\bar{t}=0$ é descrito por $\bar{t}= \pm(c / \alpha) \ln \left(\alpha \bar{x} / c^{2}\right)$, ilustrado pelas linhas tracejadas em vermelho na Fig. 8

\subsection{Cinemática de Ana segundo Bob}

No referencial $\overline{\mathcal{S}}$, Bob encontra-se em repouso em $\bar{x}=$ $c^{2} / \alpha$ e Ana, por sua vez, está em movimento. A equação da trajetória de Ana é obtida tomando-se $x=X_{\mathrm{A}} \equiv$ $k\left(c^{2} / \alpha\right)$ na segunda expressão de 23 , sendo dada por

$$
\bar{x}(\bar{t})=\frac{k c^{2}}{\alpha} \operatorname{sech}\left(\frac{\alpha \bar{t}}{c}\right) .
$$

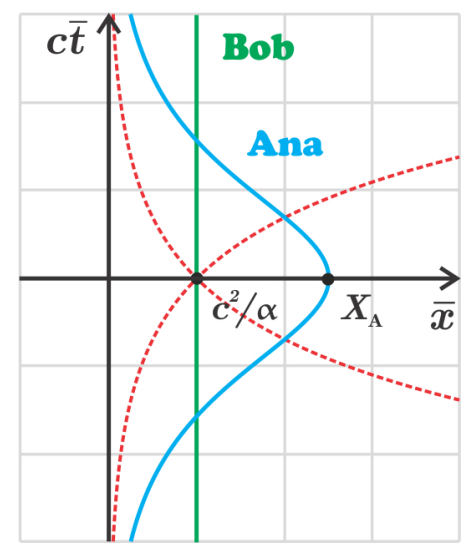

Figura 8

\footnotetext{
${ }^{24}$ Esse comportamento é análogo ao observado para raios luminosos próximos ao horizonte de eventos de um buraco negro [30]. Em particular, pode-se mostrar que o espaçotempo observado por alguém em repouso a uma certa distância do horizonte de eventos de um buraco negro de Schwarzschild é localmente indistinguível do espaçotempo observado por Bob 31].
} 
Sua velocidade é obtida de $\bar{u}(\bar{t})=d \bar{x} / d \bar{t}$,

$$
\bar{u}(\bar{t})=-k c \operatorname{sech}\left(\frac{\alpha \bar{t}}{c}\right) \tanh \left(\frac{\alpha \bar{t}}{c}\right)
$$

O primeiro ponto interessante a ser notado é que Ana descreve o movimento de Bob como uma hipérbole no espaçotempo, mas Bob não diz o mesmo sobre o movimento de Ana (Fig. 8). A razão disso é Bob observar o espaçotempo de modo diferente de Ana, como discutido na seção anterior. Pelo mesmo motivo, Bob conclui que Ana pode atingir velocidades arbitrariamente maiores que $c$ dependendo do valor de $k{ }^{25}$ No entanto, lembrando que Bob observa a luz mover-se com velocidades diferentes de $c$, enfatizamos que ele jamais observa Ana mover-se mais rápido que qualquer feixe luminoso que esteja junto a ela. Constatamos isso notando que, em cada ponto $\bar{x}$, o módulo da velocidade da luz, $|\bar{v}|=\alpha \bar{x} / c$, é sempre maior que o da velocidade de Ana, $|\bar{u}|=(\alpha \bar{x} / c) \sqrt{1-\left(\alpha \bar{x} / k c^{2}\right)^{2}}{ }^{26}$

Qual a relação entre um intervalo de tempo próprio de Ana e um intervalo de tempo do referencial de Bob? No referencial de Bob, o intervalo entre Ana sair do ponto $\bar{x}_{1}$ no instante $\bar{t}_{1}$ e chegar no ponto $\bar{x}_{2}$ em $\bar{t}_{2}$ é registrado utilizando-se dois relógios (um em cada um destes pontos). No referencial de Ana, ela está sempre em repouso, logo utiliza somente um relógio (o dela) para registrar o intervalo $\Delta \tau$ de tempo próprio. Para relacionar estes registros, notamos que o deslocamento infinitesimal $d s$ de Ana é calculado, por ela mesma, de acordo com (9) e calculado por Bob segundo (25). Como essa grandeza assume o mesmo valor em ambos referenciais, tem-se $-c^{2} d t^{2}+d x^{2}=-\left(\alpha \bar{x} / c^{2}\right)^{2} c^{2} d \bar{t}^{2}+d \bar{x}^{2}$. Ana está sempre na mesma posição em seu referencial $(d x=0)$, logo o intervalo de tempo que ela mede é próprio $(d t=d \tau)$. Isso implica na relação

$$
\Delta \tau=\int_{\bar{t}_{1}}^{\bar{t}_{2}} \sqrt{\left(\frac{\alpha \bar{x}}{c^{2}}\right)^{2}-\frac{\bar{u}^{2}}{c^{2}}} d \bar{t}
$$

a qual revela o conteúdo do lado direito da expressão (11) para o caso do MRUV relativístico. Junto às Eqs. (26) e 27), esta expressão resulta em

$$
\Delta \tau=\frac{k c}{\alpha}\left[\tanh \left(\frac{\alpha \bar{t}_{2}}{c}\right)-\tanh \left(\frac{\alpha \bar{t}_{1}}{c}\right)\right],
$$

a qual prevê a dilatação do tempo como observada por Bob. Como adotamos que o instante zero de todos relógios coincide, a relação entre os tempos medidos a partir deste instante é

$$
\bar{t}(\tau)=\frac{c}{\alpha} \tanh ^{-1}\left(\frac{\alpha \tau}{k c}\right) .
$$

\footnotetext{
${ }^{25}$ Como $k>1$ e o termo dependente de $\bar{t}$ em 27 assume valores entre $-1 / 2$ e $+1 / 2, u$ pode ser maior que $c$ para qualquer $k>2$.

${ }^{26}$ Para obter a velocidade de Ana neste formato, isole $\bar{t} \mathrm{em}$ 26. para substituí-lo em 27. Ao fim, faça uso da identidade $\tanh \left[\operatorname{sech}^{-1}(x)\right]= \pm \sqrt{1-x^{2}}$.
}

Para analisar essa relação, vamos tomar a Fig. 9 como referência e, por simplicidade, considerar intervalos medidos a partir do instante zero. Nela, a função (30) é descrita pela curva em azul. Primeiramente, Bob observa o tempo de Ana passar de modo mais rápido que o seu até o instante $\bar{t}\left(\tau^{*}\right)$, onde $\tau^{*}=(c / \alpha) \sqrt{k(k-1)}$. Após este, ele observa o tempo de Ana passar mais lentamente que o seu. Isso diz respeito ao transcorrer do tempo. A respeito dos intervalos registrados, considere o instante $\tau^{\dagger}$, solução da equação transcendental $\tau^{\dagger}=(c / \alpha) \tanh ^{-1}\left(\alpha \tau^{\dagger} / k c\right)$. Para instantes até $\bar{t}\left(\tau^{\dagger}\right)$, intervalos $\Delta \bar{t}$ registrados por Bob são menores que intervalos $\Delta \tau$ registrados por Ana e o contrário ocorre após $\bar{t}\left(\tau^{\dagger}\right)$. Da simetria do gráfico da Fig. 9 , chegamos à conclusões análogas para instantes anteriores ao zero.

A análise apresentada ilustra o quão equivocada pode ser a intuição de que "o tempo passa mais devagar para relógios em movimento" quando consideramos previsões feitas a partir de um referencial não-inercial, pois há momentos em que Bob observa o tempo transcorrer mais rapidamente para Ana, ainda que ele a observe em movimento. Além disso, vimos que intervalos de tempo registrados por Bob podem ser menores ou maiores que os registrados por Ana dependendo dos instantes abrangidos, mas, como já discutimos em outras ocasiões, tais registros podem ser questionados por outros referenciais.

Para vermos como a dilatação do tempo observada por Bob elimina a existência de um paradoxo no problema dos gêmeos, é crucial considerar registros de Ana e Bob que não sejam questionáveis. Este é o caso quando levamos em conta o registro que eles fazem, cada um com somente um relógio, para o tempo entre seus dois encontros. Segundo Ana, os encontros ocorrem nos instantes $-T$ e $T$, onde $T=(c / \alpha) \sqrt{k^{2}-1}$ (Sec. 3.3). Na próxima seção, verificaremos que estes são os valores que o tempo próprio de Ana assume no momento dos encontros. Da discussão do parágrafo anterior, se os eventos entre os dois encontros ocorrem, segundo Bob, em instantes $\bar{t}$ que satisfazem $-\bar{t}\left(\tau^{\dagger}\right)<\bar{t}<\bar{t}\left(\tau^{\dagger}\right)$, o intervalo $\Delta \bar{t}$ que ele registra é sempre menor que o registrado por Ana, garan-

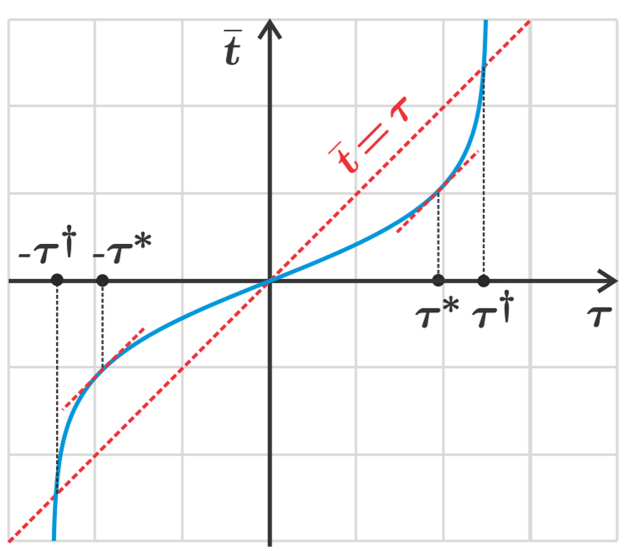

Figura 9 
tindo que não existe um paradoxo ${ }^{27}$ Para verificar que este é, de fato, o caso, é preciso mostrar que $\bar{t}(T)<\bar{t}\left(\tau^{\dagger}\right)$. Como $\bar{t}(\tau)$ é uma função de crescimento monótono 28 basta mostrarmos que $T<\tau^{\dagger}$, o que pode ser feito pela Fig. 10. Nesta, as cores azul, verde, marrom e laranja estão associadas aos casos de $k=2,3,4$ e 5 , respectivamente. Para cada valor de $k$, a curva sólida ilustra a parte positiva da função (30); a reta vertical tracejada marca, no eixo $\tau$, o valor de $T$; e, por fim, a coordenada em $\tau$ do círculo colorido corresponde ao valor de $\tau^{\dagger}$. Por esta construção, vemos que $T<\tau^{\dagger}$ para todos os casos ilustrados 29

A seguir, obteremos as expressões para as previsões de Bob para os intervalos registrados entre os seus dois encontros com Ana. Veremos que estas concordam com aquelas obtidas do ponto de vista de Ana na Sec. 3.3

\subsection{Conclusões de Bob}

Bob registra o intervalo de tempo entre seus dois encontros com Ana em $\bar{x}=c^{2} / \alpha$ e também prevê o que é registrado pelo relógio dela. Como estes dois eventos ocorrem em uma mesma posição também para Bob, ele usa somente seu próprio relógio para tal registro, garantindo que a validade desta medida não será questionada por qualquer outro referencial. Esperado pela simetria observada no movimento de Ana, os encontros ocorrem em instantes $-\bar{T}$ e $\bar{T}$ no relógio de Bob, sendo $\bar{T}$ obtido tomando-se $\bar{x}=c^{2} / \alpha$ na equação 26 para a trajetória

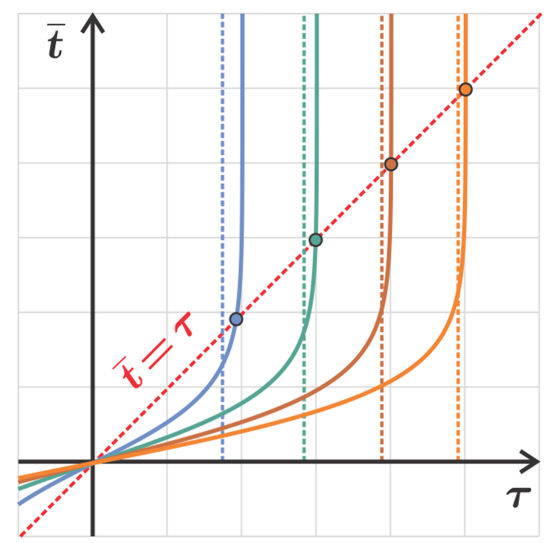

Figura 10

\footnotetext{
${ }^{27}$ É interessante notar que, mesmo entre os dois encontros com Ana, nem sempre Bob observa o transcorrer do seu tempo ser mais lento que o de Ana, pois $\tau^{*}<T$, mas a extensão desta ocorrência é suficiente para garantir que o seu intervalo registrado seja sempre menor que o de Ana.

${ }^{28}$ Falamos que uma função $f(x)$ é de crescimento monótono se ela satisfaz $f(a)>f(b)$ para todo $a>b$.

${ }^{29}$ Seria possível ter $T>\tau^{\dagger}$ para algum valor de $k>1$ não ilustrado? Não. O modo mais simples de verificar isso é construíndo-se a Fig. 10 para valores maiores de $k$. O que verifica-se é que a solução para $\tau^{\dagger}$ rapidamente tende a $k c / \alpha$ conforme o valor de $k$ aumenta por exemplo, para $k=4, \tau^{\dagger} \approx 3,997 c / \alpha$. Por outro lado, o valor de $T=(c / \alpha) \sqrt{k^{2}-1}$ aproxima-se de $k c / \alpha$ de modo mais lento, o que garante que $T<\tau^{\dagger}$ para valores de $k$ arbitrariamente grandes.
}

de Ana,

$$
\bar{T}=\frac{c}{\alpha} \operatorname{sech}^{-1}\left(\frac{1}{k}\right)=\frac{c}{\alpha} \tanh ^{-1}\left(\frac{U}{c}\right),
$$

onde a segunda igualdade vem do uso da relação $\phi=$ $\operatorname{sech}^{-1}(1 / k)=\tanh ^{-1}(U / c)$ obtida dos triângulos da Fig. 4. Com isso, vemos que o intervalo de tempo próprio registrado por Bob é igual a

$$
\Delta \bar{\tau}=2 \bar{T}=\frac{2 c}{\alpha} \tanh ^{-1}\left(\frac{U}{c}\right)
$$

o que coincide com a previsão 21 que Ana faz para o registro de Bob.

Da relação (29), temos que Bob prevê que o relógio de Ana registra o intervalo $\Delta \tau=(2 k c / \alpha) \tanh (\alpha \bar{T} / c)=$ $2 k U / \alpha$ até o segundo encontro. Essa expressão para $\Delta \tau$ não se assemelha muito a (20), pois envolve $k$. Para eliminá-lo, retornamos à Fig. 4 para notar que $\cosh \phi=$ $k=\gamma_{U}$. Com isso, finalmente, obtemos

$$
\Delta \tau=\frac{2 \gamma_{U} U}{\alpha}
$$

Lembrando-se de 20, vemos que a previsão de Bob para o intervalo registrado por Ana de fato coincide com o valor $2 T$ que ela registra.

\section{Comentários Finais}

O problema dos gêmeos é o mais antigo, e possivelmente o mais popular, da literatura de aparentes paradoxos da teoria da relatividade especial. Apesar disso, até hoje a compreensão da inexistência de um real paradoxo é, por vezes, prejudicada por certas falhas de intuição que, pelos mais diversos motivos, surgem durante o aprendizado desta teoria. Esperamos, com este trabalho, ter esclarecido algumas destas. Em particular, que (1) a relatividade especial é capaz de descrever movimentos acelerados, bem como ser usada em referenciais não-inerciais, não existindo a necessidade de invocar a relatividade geral para isso; e que (2) a intuição de que "o tempo passa de modo mais lento para relógios em movimento" geralmente falha quando o registro de tempo está sendo feito em um referencial não-inercial.

\section{Referências}

[1] A. Einstein, Ann. Phys. 17, 891 (1905).

[2] A. Einstein, $O$ ano miraculoso de Einstein: Cinco artigos que mudaram a face da física (Editora UFRJ, Rio de Janeiro, 2001).

[3] P. Pesic, Eur. J. Phys. 24, 585 (2003).

[4] H. Chang, Stud. Hist. Phil. Sci. 24, 741 (1993).

[5] R.L. Shuler Jr., J. Mod. Phys. 5, 1062 (2014).

[6] J. Crampin, W.H. McCrea, F.R.S. McNally e D. McNally, Proc. R. Soc. London, Ser. A 252, 156 (1959).

[7] E. Minguzzi, Am. J. Phys. 73, 876 (2005). 
[8] E.A. Deslogue e R.J. Philpott, Am. J. Phys. 55, 252 (1987).

[9] C.J.H. Cavalcanti e F. Ostermann, Rev. Bras. Ens. Fís. 29, 355 (2007).

[10] W. Rindler, Essential Relativity: Special, General, and Cosmological (Springer-Verlag, New York, 1977), $2^{\text {a }}$ ed.

[11] P.B. Pal, Eur. J. Phys. 24, 315 (2003).

[12] C.W. Misner, K.S. Thorne e J.A. Wheeler, Gravitation (W. H. Freeman and Company, San Francisco, 1973).

[13] M.H. Nussenzveig, Curso de Física Básica, 4: Ótica, Relatividade e Física Quântica (Blucher, São Paulo, 2014), $2^{\mathrm{a}}$ ed.

[14] D.J. Griffiths, Eletrodinâmica (Pearson, São Paulo, 2011), $3^{\mathrm{a}} \mathrm{ed}$.

[15] T. Takeuchi, An Illustrated Guide to Relativity (Cambrige University Press, New York, 2010).

[16] R. D'Inverno, Introducing Einstein's Relativity (Clarendon Press, Oxford, 1992).

[17] D. Koks, Does a clock's acceleration affect its timing rate?, Disponível em http://math.ucr.edu/home/baez/ physics/Relativity/SR/clock.html, acessado em out. 2018.

[18] J. Bailey, K. Borer, F. Combley, H. Drumm, F. Krienen, F. Lange, E. Picasso, W. von Ruden, F.J.M. Farley, J.H. Field et al., Nature 268, 301 (1977).

[19] C.E. Ross, J. Marraffino, S. Reucroft, J. Waters, M.S. Webster, E.G.H. Williams, A. Manz, R. Settles e G. Wolf, Nature 286, 244 (1980).

[20] A.M. Steane, Relativity Made Relatively Easy (Oxford University Press, Oxford, 2012).

[21] H.R. Brown, Physical Relativity: Space-time structure from a dynamical perspective (Oxford University Press, Oxford, 2005).

[22] D. Tevian, The Geometry of Special Relativity (CRC Press, Boca Raton, 2012).

[23] D. Koks, Explorations in Mathematical Physics (Springer, New York, 2006).

[24] L. Iorio, Found. Phys. Lett. 18, 1 (2005).

[25] G. Salzman e A.H. Taub, Phys. Rev. 95, 1659 (1954).

[26] Ø. Grøn, Am. J. Phys. 49, 28 (1981).

[27] E. Dewan e M. Beran, Am. J. Phys. 27, 517 (1959).

[28] J.S. Bell, Speakable and Unspeakable in Quantum Mechanics (Cambrige University Press, Cambridge, 1987).

[29] G. Muñoz e P. Jones, Am. J. Phys. 78, 377 (2010).

[30] W. Rindler, Am. J. Phys. 34, 1174 (1966).

[31] C. Semay, Eur. J. Phys. 27, 1157 (2006). 